\title{
El aprendizaje servicio universitario: materiali- zando el compromiso social de la universidad desde una educación basada en la excelencia
}

\author{
Salvador Simó ALgADo \\ Universitat de Vic \\ salvador.simo@uvic.cat
}

Recibido: $10 / 10 / 2012$

Aceptado: 23/01/2013

\begin{abstract}
Resumen
Este artículo está basado en el proyecto de Aprendizaje Servicio (ApS) (Puig, 2006) Jardín Miquel Martí i Pol, iniciado en el año 2005 en la Universidad de Vic (UVic). En el mismo, universitarios junto a personas en situación de exclusión social por motivos relacionados con la enfermedad mental, la pobreza y/o la inmigración han creado y mantenido un jardín abierto a toda la ciudadanía y dedicado al poeta Martí Pol. Es un ejemplo de cómo los campus universitarios pueden ser un vivero de proyectos que integren la excelencia en la docencia con el servicio a la comunidad en el espíritu del ApS. Pero además posee el potencial de mejorar la salud y el bienestar de los estudiantes. Sobre el proyecto Jardín Miquel Martí $i$ Pol se ha desarrollado la tesis doctoral La palabra y la acción; lucha contra la pobreza, ciudadanía y salud ocupacional mediante nuevas praxis universitarias (Simó, 2011).

Palabras clave: Aprendizaje Servicio, problema based learning (PBL), investigación, comunicación, Investigación Acción, compromiso social de la universidad.

\section{University Service Learning: Materializing Social Commitment of the University from an Education Based on Excellence}

\begin{abstract}
This article is based on the Service Learning (SL) project (Puig, 2006) Miquel Marti i Pol Garden, started in 2005 at the Universitat de Vic. In the project university students with people experiencing social exclusion for reasons related with mental illness, poverty and/or immigration have created and maintained a garden open to all citizens and dedicated to the poet Martí Pol. It is an example of how universities can be a breeding ground for projects that integrate excellence in teaching with community service in the spirit of SL. But it also has the potential to improve the health and wellbeing of students. The thesis The word and action: the fight against poverty, citizenship, and occupational health through new university praxis (Simó, 2011) has been develop based on the project.

Keywords: Service learning, problem based learning (PBL), research, communication, action research, social commitment of the university.

\section{Referencia normalizada}

SIMÓ ALGADO, Salvador (2013): "El aprendizaje servicio universitario: materializando el compromiso social de la universidad desde una educación basada en la excelencia". Estudios sobre el mensaje periodístico. Vol. 19. Núm. especial abril, págs.: 1027-1036. Madrid, Servicio de Publicaciones de la Universidad Complutense.
\end{abstract}

Sumario: 1. Introducción; 1.1. Justificación. 2. Metodología. 3. El proyecto Jardín Miquel Martí Pol; 3.1. Investigación; 3.2. El jardín como espacio de encuentro con el Otro; 3.2.1. Aprendizaje; 3.2.2. Escuela de ciudadanía; 3.2.3. Bienestar de los estudiantes. 4. Conclusiones. 5. Referencias bibliográficas. 


\section{Introducción}

Como docentes tenemos que preparar a los estudiantes para la sociedad del riesgo (Beck, 1998). Vivimos inmersos en una modernidad líquida (Bauman, 2005), marcada por declinar de la ciudadanía y una grave crisis económica, ecológica y social. El paro convive con una precariedad laboral, en un proceso de brasileñización del mercado laboral europeo (Beck, 2000). Un trabajo que pierde sus referentes éticos y provoca la corrosión del carácter (Sennett, 2006). La globalización económica no ha venido acompañada de una globalización de los derechos humanos (DH). Los ciudadanos afrontan estos riesgos desde la soledad, sin la protección de un Estado del Bienestar en proceso de desmantelamiento (Bourdieu, 1999).

Como bien dice Boff (2000) el sufrimiento más que la admiración hace pensar. En este contexto Said (2006) cuestiona el rol de los intelectuales que por interés callan, cometiendo un delito de silencio (Mayor, 2006). Ante este escenario la Universidad, en el marco del nuevo Espacio Europeo de Educación Superior (EEES), debe erigirse como motor de la sociedad civil y abordar con valentía los retos globales de nuestra época. Debemos formar profesionales capaces de trabajar en equipos multidisciplinares, desde una comprensión de la compleja problemática actual, con las competencias para su mejora. El objetivo no se limita a comprender el mundo, sino a transformarlo (Marx, 1988).

\subsection{Justificación}

El proyecto surgió de un análisis crítico del contexto educativo. Los documentos oficiales expresan la determinación de crear una Universidad comprometida con la sociedad, al servicio de un desarrollo humano más armonioso, para hacer retroceder la pobreza, la exclusión y las guerras (UNESCO, 2002; Delors, 1996). En La Carta Magna de las Universidades (Martín, 1999) los rectores de las universidades europeas, consideraron que el futuro de la Humanidad dependía en gran medida de las universidades. Harvaky (2006) analiza los objetivos fundacionales de las universidades, donde se considera la educación como la base de todo desarrollo democrático, con el servicio a la sociedad como propósito principal. Pero la Universidad enfrenta una serie de obstáculos como son: 1) El platonismo: La teoría platónica de la educación era elitista e idealista. El objetivo era la ciencia pura. 2) La comercialización: La guerra fría aceleró la comercialización de las universidades. 3) El etnocentrismo de las disciplinas que impide la cooperación interdisciplinaria.

¿Estamos preparando a los estudiantes para el nuevo orden mundial? Sabemos, basados en las evidencias científicas que debemos actuar, y las universidades deberían estar concienciando a los universitarios y a la comunidad de los peligros existentes, y planificando estrategias para el cambio. Pero las universidades muestran negación e inercia. En general, el currículo no recoge temas globales, como la crisis ecológica o social (Thibeault, 2006).

Es preciso preparar a los estudiantes de salud y sociales para la compleja realidad contemporánea. En el aula los mismos se quedan con diagnósticos como esquizofrenia, sin ver que forman parte de una persona, que más allá de su enfermedad tiene un 
potencial, unos valores, etc. Por ello se vio la necesidad de crear un espacio de encuentro con el Otro, inspirados en la ética de Lévinas (2000).

\section{Metodología}

La Universidad debe asumir su responsabilidad como institución clave de la sociedad civil, entre el Estado y el mercado (Barber, 2000), como garante de la democracia, la justicia social y la libertad. Una Universidad que denuncie las realidades intolerables y que anuncie nuevas utopías posibles. Se impone la educación para una ciudadanía cosmopolita, arraigada en el local, inspirados por Kant (1946) quien soñaba con la unificación perfecta de la especie humana a través de una ciudadanía común. Una ciudadanía que pueda desarrollarse en los diferentes escenarios post-nacionales que se nos plantean, en forma de realidad europea (Habermas, 1999), o sociedad civil global (Castells, 2005). La educación ciudadana impulsa el sentido de pertenencia, la cohesión social y la cultura de los DH y la sostenibilidad, e integra la responsabilidad personal con el destino colectivo de la Humanidad (Paz Abril, 2007). Los estudiantes precisan entender el contexto que determina sus vidas. Leer el mundo es un acto anterior a la lectura de la palabra, ya que no existe una práctica educativa indiferente a propósitos sociales y políticos (Freire, 2003).

Siguiendo el trabajo de Manzano (2010) podemos afirmar que el modelo de ApS integra la teoría con la práctica, la formación con el compromiso, la cognición con la emoción. El ApS orienta los logros académicos hacia la construcción de ciudadanía a través del servicio comunitario. Genera un crecimiento intelectual y personal, junto a un compromiso social (Ngai, 2006), transmitiendo valores de justicia social. Es un programa de servicio solidario orientado a atender necesidades de una comunidad, y planificado de forma integrada con los contenidos curriculares (Ramson, 2009). Eyler (2003) destaca los efectos en los estudiantes de tipo personal (eficacia personal, identidad, desarrollo moral), social (reducción de estereotipos, responsabilidad social, habilidades ciudadanas, compromiso), y de aprendizaje (resultados académicos, aplicación de aprendizajes en el mundo real, pensamiento crítico, análisis de problemas, comprensión de la complejidad).

Este aprendizaje con un alto contenido ético tiene más sentido en el marco de una sociedad hedonista que reduce al bios politikos a un homo consumens, marcada por una ética laxa que conduce al ocaso del deber (Lipovetsky, 1994). Parece como si aquellos valores que hacen habitable el mundo se van descomponiendo, bajo la acción del fermento del consumismo y del individualismo. Como indicó Kant (1991) hemos de educar pensando en la mejor situación posible en el futuro. Es tiempo de educar en virtudes como la empatía y la compasión. Tiempo para forjar el carácter de los estudiantes desde la ética. No se puede transformar el mundo desde la desmoralización, sino desde personas que pasan de espectadores a actores morales.

\section{El proyecto de ApS Jardín Miquel Martí i Pol}

El proyecto, actualmente vigente, se inició en 2005 en la UVic. En el mismo estudiantes han trabajado con personas en situación de exclusión social en la creación y mantenimiento de un jardín situado en el campus y abierto a toda la ciudadanía. Las 
sesiones se desarrollan durante 3 horas una vez a la semana. Es una sinergia entre las instituciones políticas, empresariales y civiles ${ }^{1}$, manifestando la necesidad de desarrollar alianzas estratégicas. El proyecto tiene las siguientes dimensiones:

- Construcción de comunidades inclusivas y sostenibles: Definidas como "aquel grupo humano que se reconoce interdependiente y en el que toda persona se siente y participa como ciudadano de pleno derecho, a pesar de cualquier problemática, y que se desarrolla en armonía con el medio natural asegurando las necesidades presentes sin comprometer las de las generaciones futuras para satisfacer las propias" (Simó, 2012, Pág.13). El objetivo es la integración laboral así como desarrollar un trabajo de concienciación de la comunidad, para romper los prejuicios y el estigma. Por ello es básica la política de comunicación, por lo que se han desarrollado conferencias, exposiciones y el uso de las TIC (blog ${ }^{2}$, grupo facebook ${ }^{3}$, etc.).

- Salud: Implica un completo estado de bienestar físico, psíquico y social (WHO, 1946). La jardinería se realiza al aire libre, en contacto con la tierra, lo que favorece la capacidad de introspección y un sentimiento de relajación. Es un espacio social, e implica la realización de ejercicio físico. Es una experiencia estética que roza el arte.

- Educativa: Estudiantes de terapia ocupacional (TO) han participado ${ }^{4}$, junto a estudiantes de educación, arte y de ciencias de la comunicación, colaborando en la política de comunicación, desarrollando un aprendizaje basado en problemas (ENOTHE, 2004).

- Ecológica: Es trascendental dar una educación ecológica. Según Boff(2000) la ecología es la relación del todo con el todo, así el principal problema ecológico es la pobreza. La educación debe ayudar a entender la interconexión de todos los seres de la biosfera. El proyecto actual se amplía con la reforestación de espacios naturales.

El proyecto Jardín Miquel Martí i Pol ha sido reconocido a nivel nacional e internacional. El año 2010 fue seleccionado como una buena praxis de ApS por la Fundación Bofill. El mismo año fue reconocido como Example of good praxis in the fight against poverty, por tribuna del proyecto Competences for Poverty Reduction, financiado por la Unión Europea.

${ }_{1}$ Las intituciones participantes son políticas (Ajuntament de Vic, Consell Comarcal d'Osona), empresariales (Rotary Club, Funcació Girbau, Fundació La Caixa), y civiles (Caritas Arxipestal, Fundació Centre Mèdic Psicopedagògic d'Osona).

2 Ver http://www.jardimiquelmartipol.blogspot.com.es/

3 Jardí Miquel Martí Pol.

${ }^{4}$ Han participado estudiantes de $1^{\circ}$ de diplomatura a través de la asignatura Actividades Ocupacionales Aplicadas, como estudiantes de $2^{\circ}$ y $3^{\circ}$ como voluntarios. Desde 2009 participan estudiantes del grado de T.O. en el nuevo EEES. 


\subsection{Investigación}

Sobre el proyecto se ha desarrollado una investigación (Simó, 2011) basada en un paradigma crítico hermenéutico. Las realidades que nos causan indignación deben llevarnos a cuestionar críticamente la naturaleza y condición moral de nuestra sociedad, y a emprender una búsqueda de alternativas, teóricamente fundamentadas (Marcuse, 1968). La idea de la hermenéutica es que en todo obrar humano hay una posibilidad de comunicación y comprensión (Escalante, 2002). El ser humano es una unidad narrativa (Ricoeur, 1999). Para esta comprensión es vital la capacidad de escuchar a colectivos muchas veces silenciados, como las personas en situación de exclusión social o los propios estudiantes, para desarrollar una sociología de las emergencias (Sousa, 2005).

Se ha desarrollado una Investigación Acción (IA), cuya finalidad es generar un conocimiento transformador. Así se combinan los ciclos de acción con los de reflexión, que Mounier (2002) comparaba con los movimientos de sístole y diástole del corazón humano. La IA es un proceso por el cual se examinan sistemáticamente cuestiones desde las experiencias vividas por los miembros de la comunidad. Esta filosofía es cercana a Dewey (1969) quien afirmaba la necesidad de que el profesor tenga una comprensión del mundo para transformarlo.

La metodología desarrollada han sido las entrevistas en profundidad y la realización de diarios compartidos por parejas formadas por un estudiante y un usuario del proyecto. A continuación aparecen los temas surgidos y una pequeña muestra de las narrativas de los estudiantes. El resto de narrativas, así como las historias de vida realizadas con los usuarios, pueden consultarse on line ${ }^{5}$.

\subsection{El jardín como espacio de encuentro con el Otro}

"Participar en el proyecto ha sido de las mejores cosas que me han pasado. Me ha dado la oportunidad de conocer a unas personas magníficas que día tras día me enseñan multitud de cosas" (Iván). Este encuentro permite trascender los prejuicios: "Los jardineros ni son incapaces ni responden a los prejuicios que tiene la sociedad" (María); "En el jardín no hay diagnósticos" (Oscar). Se genera un sentimiento de pertenencia al grupo: "Cuando trabajo en el jardín siento que soy parte de un grupo, un espacio que ha sido producto de mucho esfuerzo. Detrás del mismo hay una cadena de personas que han visto más allá de la tierra vacía y estéril. Han tenido un sueño y lo han llevado a cabo. Me siento orgullosa de formar parte de esta cadena que da continuidad al proyecto" (Laia).

\subsubsection{Aprendizaje}

"Me considero afortunado de haber podido ser partícipe de un aprendizaje en un proyecto de una extremada belleza" (Christian); "Me ha dado la oportunidad de conocer la verdadera esencia de la TO" (Iván). Los estudiantes se sienten empoderados: "Me dio la oportunidad de creer en mi propio potencial y en el de la unión de un grupo

5 La tesis doctoral está disponible en Tesis Doctorals en Xarxa: http://www.tesisenred.net/handle/10803/9325 
de personas. Aprender a comunicarme, creer que soy capaz de hacer algo hermoso. Me convenció de que es posible cambiar, yo mismo y la sociedad" (Oscar); "Me ha ayudado a comprender la realidad social actual, conceptos como ciudadanía, participación, estigma, comunidad, educación..." (Oscar). Este contacto conciencia de la exclusión social: "La exclusión social es uno de los peores castigos que se le puede dar a una persona, somos interdependientes, construimos nuestra identidad a través de los otros. Es un atentado contra el espíritu humano" (Christian).

\subsubsection{Escuela de ciudadanía}

"Como ciudadana me siento más comprometida y tengo más ganas de participar activamente para intentar mejorar el futuro de nuestra sociedad. Los ciudadanos unidos podemos llevar a cabo grandes proyectos para mejorar la comunidad" (Laia); "Me ha servido para tener presente que tengo unos derechos como ciudadano y unos deberes" (Oscar); "No existe una relación paternalista donde prevalece la enfermedad, existe una relación entre personas libres con capacidad de transformar el mundo" (Laia); "Ciudadanía implica participación, capacidad de acción, y transformación. Marx hablaba sobre el zoon politikon, un hombre político capaz de actuar en el mundo material y real" (Christian). Ciudadanía consciente de la problemática ecológica: "Me convirtió en un jardinero, ahora cuido la Tierra, planto semillas y saboreo los frutos que me regala el milagro de la vida" (Oscar).

\subsubsection{Bienestar de los estudiantes}

Se promueve un bienestar físico: "Soy una persona muy activa, me ayuda a sentirme viva y sentir que estoy aprovechando el día" (María). Un bienestar mental: "Trabajar en el jardín me aporta tranquilidad, un espacio de reflexión y conexión con la naturaleza. Agradezco mucho el silencio, el olor de la tierra, el perfume de las flores, el sonido del agua, la belleza del jardín, porque me aíslo ante los problemas" (Laia). "Podías desarrollar un trabajo de conocimiento personal donde mi yo interior floreció bello y sano" (Oscar). Un bienestar social: "Estás a gusto con las personas y sientes el respeto que hay en todo momento" (María). Un espacio que ayuda a superar situaciones difíciles: "El año pasado fue un año difícil por una situación personal y familiar y el hecho de tener cosas que hacer durante el día hacía que me olvidara por momentos" (María). Y facilita dejar el consumo de drogas: "Mi adicción al hachís se convirtió en una lucha a la cual me enfrenté gracias al jardín. Me hizo darme cuenta de como estando ocupado en un lugar agradable, podía ser capaz de conseguir que mi mente se sintiera tranquila y feliz. Me hizo sentir valiente para enfrentarme a mí mismo" (Oscar).

En el diario compartido por Christian y Plácido podemos leer este fragmento que captura el potencial del ApS: "A lo largo de este año hemos estado creciendo juntos, en la cotidianidad de cada día. Con cuidado y delicadeza hemos construido una experiencia conjunta y sincera. La naturaleza hace ceder muros, hacia un espacio silencioso, un lugar donde hay cabida para cada uno de nosotros. Cada paso dentro de esta relación puede ofrecer comprensión y conciencia sobre elementos que quizá de otro modo pasarían inadvertidos. Estar en un espacio durante un tiempo hace que nuestras 
experiencias, emociones, sentimientos y pensamientos queden escondidos en varios rincones del lugar. Poder ir haciendo un registro ha sido una hermosa experiencia, ya que ha puesto de relieve la necesidad de interactuar y de compartir".

\section{Conclusiones}

¿Cómo desarrollar proyectos de ApS en el marco del nuevo EEES? En el proyecto han participado desde 2005 más de 50 estudiantes. Esta metodología se puede integrar en el marco de diferentes asignaturas o en el contexto de los 6 créditos optativos RAC (Reconocimiento de actividades culturales y de cooperación). Supone una nueva praxis educativa que garantiza la adquisición de competencias, generando nuevos espacios docentes. Además responde a las amenazas manifestadas por la institución universitaria: el platonismo, al ser una praxis inmersa en el mundo real; el etnocentrismo disciplinario, al poder ser lugar de encuentro de diferentes saberes; y la comercialización, al prestar un servicio a los sectores más vulnerables.

Cuando a través de esta estrategia los estudiantes aprenden valores no sólo se están formando para una ciudadanía comprometida. Los valores son el mapa que nos ayudan a guiar en nuestra vida y a dar un sentido a nuestra existencia. Esta experiencia de sentido es el antídoto a graves problemas sociales como la depresión, la agresión y la adicción (Frankl, 1964). Nuestros estudiantes no escapan a estas problemáticas, en especial la adicción a las drogas. El proyecto ha mostrado su impacto positivo en la salud de los estudiantes.

¿Cómo integrar la investigación con los proyectos para desarrollar una praxis basada en la evidencia? Dentro de un paradigma hermenéutico y crítico, hemos desarrollado una IA que está relacionada con los principios de participación y democracia.

¿Cómo podemos crear comunidades más saludables e inclusivas? La Universidad debe recuperar su compromiso con la sociedad, en especial con los sectores más vulnerables. El proyecto muestra nuestra capacidad de crear comunidades inclusivas y sostenibles. Por eso es necesaria una alianza estratégica, entre la sociedad civil, el sector público y empresarial. Ante una realidad compleja se impone una comprensión e intervención complejas (Morin, 2002), que supere el etnocentrismo disciplinar. Una intervención basada en el empoderamiento de los estudiantes, reconociendo su potencial y sus capacidades, para que puedan controlar su propia vida (Sen, 2000). En un momento de crisis como el actual no nos podemos permitir como sociedad el inmenso capital humano e intelectual que hay inactivo en la Universidad.

El proyecto es una metáfora de lo que podemos hacer a nivel de la sociedad. El sustituir una sociedad competitiva, atomista, basada en el darwinismo social y la exclusión de grandes sectores de la población, por una comunidad inclusiva, basada en el reconocimiento mutuo como seres interdependientes. En una época gris como la nuestra, donde la crisis actual parece que nos atenaza el alma, debemos recordar que la actividad humana es nuestra capacidad de poder empezar que los griegos llamaban archein. Somos seres capaces de palabra, que denuncie las injusticias presentes, y de acción, para crear nuevas utopías, desde una fenomenología del hombre capaz (Ricoeur, 2005). De la misma manera que en Italia de los siglos XV y XVI, estamos llamados a protagonizar un nuevo Renacimiento. Es tiempo de reforestar el mundo de los va- 
lores que lo hacen habitable. Como docentes no tenemos tiempo que perder. Debemos recuperar la sana capacidad de indignación ante la injusticia: nos rebelamos, por lo tanto existimos (Camus' 2002). Un sentimiento de rebeldía que ponga en marcha el viento del pensamiento y el milagro de la acción humana (Arendt, 1997). Porque como afirmaba el poeta Martí Pol(1999) "todo está por hacer, pero todo es posible".

\section{Referencias bibliográficas}

ARENDT, Hanna (1997): ¿Qué es la política? Barcelona, Paidós.

BARBER, Benjamín (2000): Un lugar para todos. Barcelona, Paidós.

BAUMAN, Zygmunt (2005): Vidas desperdiciadas. La modernidad y sus parias. Barcelona, Paidós.

BECK, Ulrich (1998): La sociedad del riesgo. Barcelona, Paidós.

BECK, Ulrich (2000): Un nuevo mundo feliz. Barcelona, Paidós.

BOFF, Leonardo (2000): La dignidad de la tierra. Madrid, Trotta.

BOURDIEU, Pierre (1999). Cortafuegos. Barcelona: Anagrama.

CAMUS, Albert (2002): El hombre rebelde. Barcelona, Alianza editorial.

CASTELLS, Manuel (2005): Global governance and global politics. En Political Science and Politics, Vol. 38, nº1, págs. 9-15.

DELORS, Jacques (1996): La educación encierra un tesoro. Barcelona, UNESCO.

DEWEY, John (1969): The ethics of democracy. Carbondale, Southern Illinois University Press.

ENOTHE (2004): Occupational Therapy education in Europe: PBL Stories. Amsterdam, ENOTHE.

ESCALANTE, Fernando (2002): "Hermenéutica y ciencias sociales". En ALTAMIRANO, Carlos: Términos críticos de sociología de la cultura. Barcelona, Paidós.

EYLER, J.S. (2003): "What We Know About the Effects of Service-Learning”. En Introduction to Service-Learning Toolkit. Second Edition. Providence, RI, Brown University.

FRANKL, Victor (1964): El hombre en búsqueda de sentido. Barcelona, Herder.

FREIRE, Paulo (2003): El grito manso. Buenos Aires, Siglo XXI.

HABERMAS, Jürgen (1999). La inclusión del otro. Barcelona, Paidós.

HARKAVY, Ira (2006): "The role of the universities in advancing citizenship and social justice". En Education, citizenship and social justice. 1 (1). Disponible en: http://esj.sagepub.com. [Consultado: 5 de enero de 2007]

KANT, Immanuel (1946): Lo bello y lo sublime. Madrid, Espasa Calpe.

KANT, Immanuel (1991): Sobre pedagogía. Vic, Eumo Editorial.

LÉVINAS, Emmanuel (2000): Ética e infinito. Madrid, La balsa de la medusa. 
LIPOVETSKY, Gilles (1994): El crepúsculo del deber. Barcelona, Anagrama.

MARTI POL, Miquel (1999): Antología Poética. Barcelona, Edicions 62.

MARCUSE, Herbert (1968): El hombre unidimensional. Barcelona, Seix Barral.

MARTÍN, Lorenzo (1999): “Carta magna de las universidades europeas". En Revista de administración pública, Vol. 118, págs. 469-474.

MARX, Karl. (1988): Textos cardinales. Barcelona, Ediciones Península.

MAYOR ZARAGOZA, Federico (2006): "Delito de silencio". En El País, publicado el 30 de noviembre de 2006.

MANZANO, Vicente (2010): “El APS y su potencial para la educación superior”. En VI Jornadas de educación en Psicología. Sevilla, Universidad de Sevilla, 15 de junio de 2010.

MORIN, Edgar (2002): Los siete saberes necesarios para la educación del futuro. Barcelona, Paidós.

MOUNIER, Emmanuel (2002): El personalismo. Salamanca, Ediciones Sígueme.

NGAI, Steven (2006): "Service-learning, personal development, and social commitment”. En Adolescence, Vol. 41, nº161, págs. 165-176.

PAZ ABRIL, Demetrio de (2007): Escuelas y educación para la ciudadanía global. Barcelona, Intermon Oxfam.

PUIG, Josep María (2006): Aprenentatge Servei. Educar per a la ciutadania. Barcelona, Octaedro.

RANSOM, Lillie (2009): "Sowing the seeds of citizenship and social justice". En Education, citizenship and social justice, Vol. 4, págs. 211-224.

RICOEUR, Paul (1999): Historia y narratividad. Barcelona, Paidós.

RICOEUR, Paul (2005): Caminos del reconocimiento. Madrid, Editorial Trotta.

SEN, Amartya (2000): Desarrollo y libertad. Barcelona, Planeta.

SENNETT, Richard (2006): La corrosión del carácter. Barcelona, Anagrama.

SAID, Edward (2006): Representaciones del intelectual. Barcelona, Paidós.

SIMÓ ALGADO, Salvador (2011): La palabra y la acción. 2011. Tesis doctorals en xarxa. Disponible en http://tdx.cat/bitstream/handle/10803/9325/PALABRAACCION.pdf? sequence $=1$

SIMÓ ALGADO, Salvador (2012): “Terapia Ocupacional eco-social”. En Cadernos Terapia Ocupacional. UFSCar, São Carlos, Vol. 20, nº 1, págs. 7-16.

SOUSA, Boaventura (2005): El milenio huérfano. Madrid, Editorial Trotta.

THIBEAULT, Rachel (2006): "Globalization, universities and the future of occupational therapy”. En Australian Journal of Occupational Therapy, Vol. 53, pág. 159165 . 
UNESCO (2002): “Constitución de la UNESCO". Disponible en: http://portal.unesco.org/es/ev.phpURL_ID=15244\&URL_DO=DO_TOPIC\&URL SECTION=201.html. [Consultado: 10 de marzo de 2010]

WHO (1946): Constitution of the World Health organization. New York, WHO.

\section{Salvador SIMÓ ALGADO}

Universidad de Vic

Departamento de Salud y Acción Social

Doctor en Educación Inclusiva y profesor salvador.simo@uvic.cat 